PSS PROCEEDINGS

\title{
Gravitational wave astronomy: now and future
}

\author{
Alicia M. Sintes* \\ Departament de Física, Universitat de les Illes Balears, Cra. Valldemossa Km. 7.5, E-07122 \\ Palma de Mallorca, Spain. \\ Max-Planck-Institut für Gravitationsphysik, Albert Einstein Institut, Am Mühlenberg 1, D-14476 \\ Golm, Germany. \\ E-mail: sintes@aei.mpg.de
}

This paper briefly summarizes the status of operating gravitational wave facilities, plans for future detector upgrades and the status of the space-based gravitational wave detectors like LISA. It also describes some of the most promising sources of gravitational waves for these ground and space based detectors.

Supernovae: lights in the darkness (XXIII Trobades Científiques de la Mediterrània) October 3-5 2007

Maó, Menorca, Spain

${ }^{*}$ Speaker. 

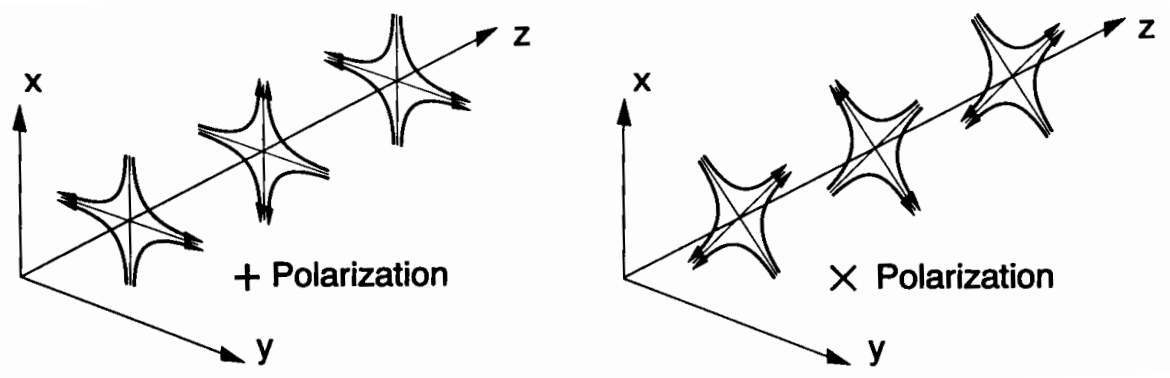

Figure 1: Gravitational waves have two polarizations. These are vertical-horizontal, like an addition symbol $"+"$ and at 45 degrees to these directions, like an " $\times$ ". A small mass placed at various points along the gravitational wave shown above will be stretched along one axis and squeezed along the perpendicular axis, then one-half cycle later the reverse occurs and the axis that was squeezing now stretches while the stretching axis squeezes.

\section{Introduction}

Gravitation governs the large scale behavior of the Universe. According to Einstein's theory of general relativity, gravity can be expressed as a space-time curvature $[1,2]$. One of the theory predictions is that a changing mass distribution can create ripples in space-time that propagate away from the source at the speed of light, carrying energy and also information about the astrophysics of the source. These freely propagating ripples in space-time are called gravitational waves.

Gravitational waves have the effect of stretching and contracting space-time. Like its electromagnetic counterpart, the effect of gravitational radiation is transverse to the direction of propagation and also has two polarizations (see figure 1). Because gravity is very weak, compared with other fundamental forces, the gravitational waves we expect to observe must be emitted by massive objects undergoing large accelerations.

The prediction of gravitational waves is one of the most profound differences between Einstein's theory of general relativity and the Newtonian theory of gravity that it replaced. The confirmation of this conjecture would be fundamental on its own. Moreover gravitational waves would provide us with information on strong field gravity, the untested domain of strongly curved spacetime, where Newtonian gravity is no longer even a poor approximation.

Any attempts to directly detect gravitational waves have not been successful yet. However, their indirect influence has been measured in the binary neutron star system PSR 1913+16, discovered in 1974 by Russell Hulse and Joe Taylor [3, 4, 5]. This system consists of two neutron stars orbiting each other. One of the neutron stars is active and can be observed as a radio pulsar from earth. Since the observed radio pulses are Doppler shifted by the orbital velocity, the orbital period and its change over time can be determined precisely. If the system behaves according to Einstein's theory, it will loose energy through the emission of gravitational waves. As a consequence the two neutron stars will decrease their separation and orbiting around each other at a higher frequency. From the observed orbital parameters one can first compute the amount of emitted gravitational waves and then the inspiral rate. The calculated and the observed inspiral rates agree within exper- 
imental errors, to within about $0.2 \%$ [6].

Gravitational waves are quite different from electromagnetic waves. Electromagnetic waves are easily scattered and absorbed by dust clouds between the object and the observer, whereas gravitational waves will pass through them almost unaffected. This gives rise to the expectation that the detection of gravitational waves will reveal a new and different view of the universe. In particular, it might lead to new insights in strong field gravity by observing black hole signatures, large scale nuclear matter in neutron stars, inner processes in supernova explosions, and, of course, the possibility to discover new kinds of astrophysical phenomena, from our own Galaxy up to cosmological distances.

Gravitational waves come in many different frequencies. But unlike electromagnetic waves, it is not the microscopic processes deep inside the sources that determine the wavelength, but the global properties of the sources, leading to wavelengths of the order of the source sizes. These range from $10^{-17} \mathrm{~Hz}$ in the case of ripples in the cosmological background, through signals in the audio band from the formation of neutron stars in supernova explosions, to $10^{10} \mathrm{~Hz}$ from the cosmological background itself. The study of the full diversity of gravitational waves, in the different frequency bands, requires complementary approaches that include: cryogenic resonant bar detectors, laser interferometer detectors on earth and in space, Doppler tracking of spacecraft, timing of millisecond pulsars, etc. For an overview of frequency bands, detection methods and sources see [7], and references therein.

The most predictable sources are binary star systems. However there are many sources of much greater astrophysical interest associated with black hole interactions and coalescences, neutron stars coalescences, low-mass X-ray binaries, such as Sco-X1, stellar collapses to neutron stars and black holes (supernova explosions), rotating asymmetric neutron stars such as pulsars, and the physics of the early universe. The signals from all these sources are at a level where detectors of very high strain sensitivity - of the order of $10^{-22}$ to $10^{-23}$ over relevant timescales - will be eventually required to allow a full range of observations and such detectors may be on ground or in space.

The direct detection of gravitational waves has been subject of some controversy ever since their prediction by Einstein. However with the commissioning, operation and potential upgrading of the long baseline detectors LIGO, GEO600, VIRGO and TAMA, we are heading into an era where this controversy will be resolved [8]. These laser interferometer detectors incorporate high power stabilized laser sources, complicated optical configurations, suspended optical components and high performance seismic filters. They have arm lengths of up to several $\mathrm{km}$ and operate in a ultra high vacuum environment.

\section{The search for gravitational waves}

\subsection{Resonant mass detectors}

The development of gravitational wave detectors has been a long process, dating to the work of Joseph Weber in the 1960s $[9,10]$. Stimulated by predictions of the possibility of earth-incident gravitational waves with amplitude of order $10^{-17}$ at frequencies near $1 \mathrm{kHz}$, Weber set out to build a detector sufficiently sensitive to observe them. His idea was to use an aluminum bar of dimension two meter long, and one-half meter diameter, whose resonant mode of oscillation $(\sim$ 


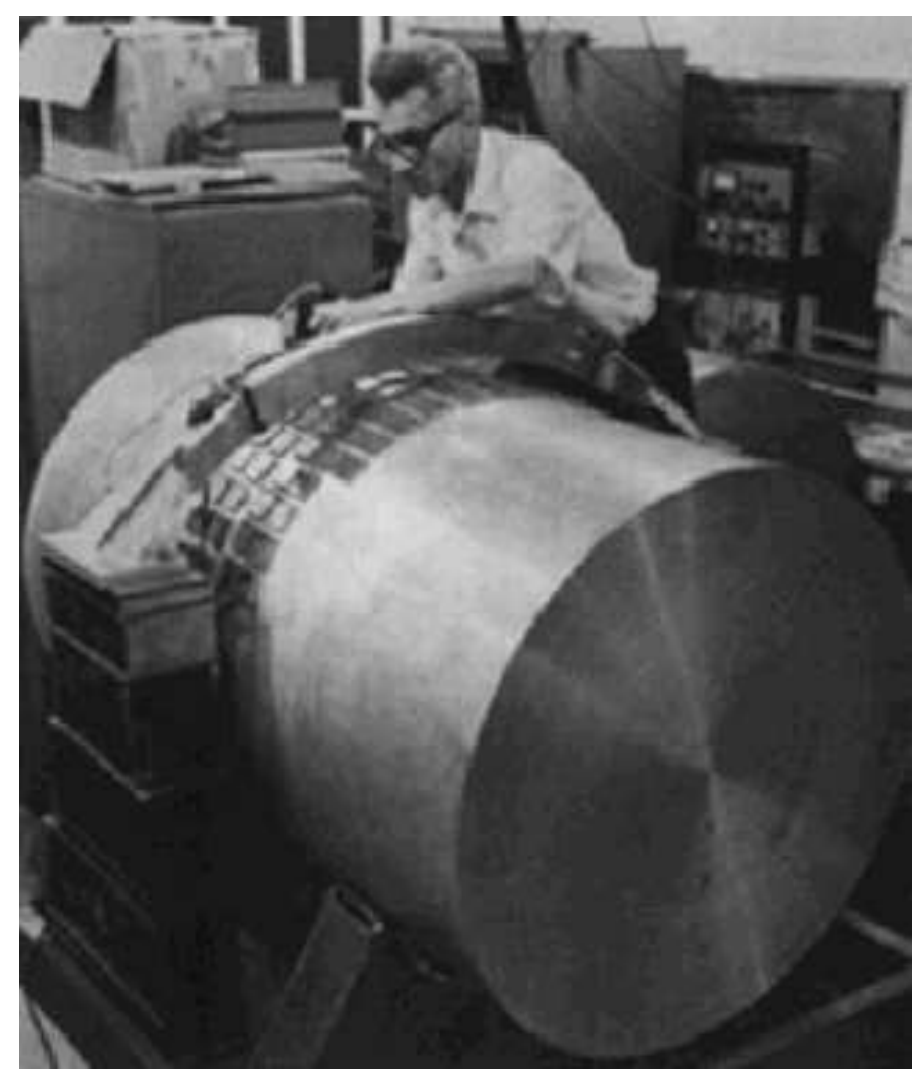

Figure 2: Joseph Weber at the University of Maryland with the first resonant gravitational wave detector.

$1.6 \mathrm{kHz}$ ) would overlap in frequency with the incoming waves. The bar, built at the University of Maryland (see figure 2), was fitted with piezoelectric transducers to convert the bar motion to an electrical signal, and provided a strain sensitivity of order $10^{-15}$ over millisecond time scales.

Over the ensuing decades, sensitivity has been improved by several orders of magnitude. Cryogenic technology has reduced mechanical thermal noise, and also allowed the use of ultra-quiet SQUID amplifiers. New vibration isolators and transducers have also played an important role in the achievement of rms strain sensitivities of about $10^{-19}$. The AURIGA detector [11] is one of this latest generation of resonant-mass detectors. It is located in Legnaro. The Rome group operates two comparable detectors, NAUTILUS in Frascati and EXPLORER at CERN [12]. The Louisiana State group operates ALLEGRO on the LSU campus in Baton Rouge [13].

Resonant-mass detectors have their sensitivity concentrated in a band around the bar's resonant frequency, or in a pair of such bands around the coupled resonances of the bar-transducer system. The current generation exhibits sensitivity of about $10^{-21} / \sqrt{\mathrm{Hz}}$, in bands measuring tens of Hertz wide.

\subsection{Ground based interferometers}

With the increasing theoretical confidence that gravitational wave strains were likely to be of the order of $10^{-21}$ or less and could encompass a wide range of frequencies, experimentalists 


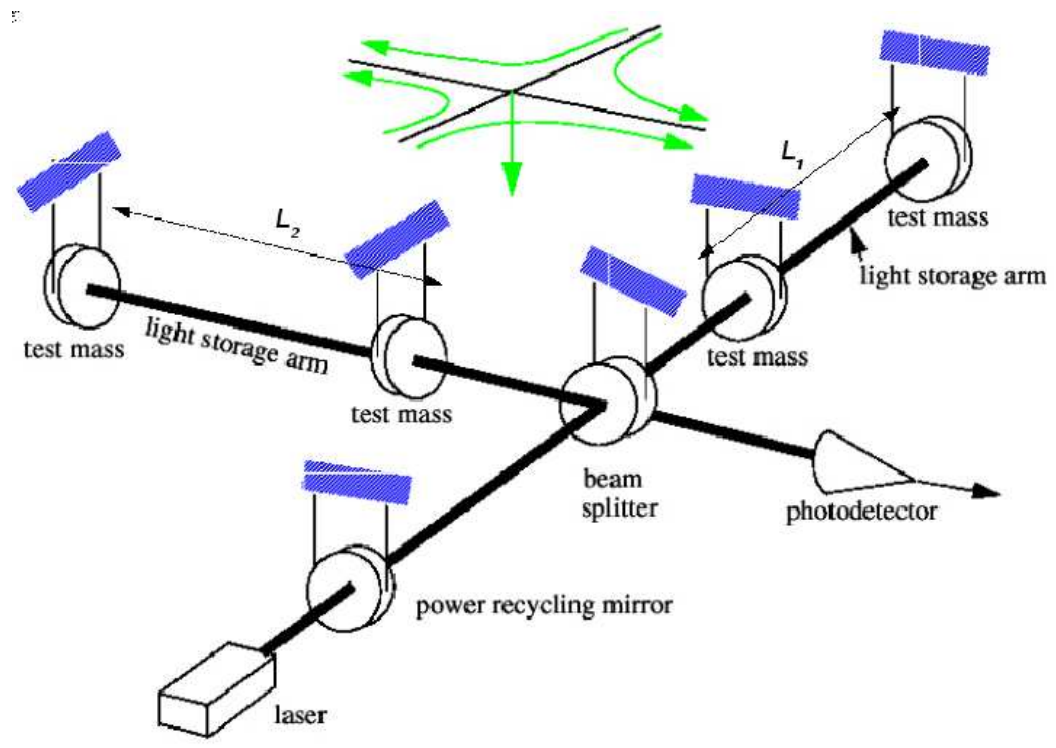

Figure 3: Optical topology used by the laser interferometer gravitational wave detectors: a Michelson interferometer with Fabry-Perot cavities in the arms. A passing gravity wave will stretch one arm and contract the other. The mirrors are suspended by wires like a pendulum; this allows free motion when a gravity wave passes and also provides isolation from seismic noise.

sought a more sensitive and wider-band means of detection. Such a means became possible with the development of the laser interferometer, first proposed by Forward [14], and independently, by Weiss [15]. This device used the configuration of the Michelson interferometer to achieve differential sensitivity to the instrument arm length changes caused by an incident gravitational wave (see figure 3). The key idea was that the speed of light (accessed in an interferometer), as compared to the much slower speed of sound (accessed in a bar), allowed for the implementation of a greatly increased measurement pathlength over a cycle of a gravitational wave, which offered correspondingly greater strain sensitivity.

The first working laser interferometer, built by Forward [16], was $2 \mathrm{~m}$ in arm length and achieved $10^{-16}$ strain sensitivity in a $1 \mathrm{~Hz}$ bandwidth at $1 \mathrm{kHz}$. Subsequent advanced versions, using improved laser stability, optics, and isolation from background seismic noise, were built at Caltech [17], University of Glasgow [18], and Garching [19]. They were 40, 10 and $30 \mathrm{~m}$ in length and achieved strain sensitivities at several hundred $\mathrm{Hz}$ in a $1 \mathrm{~Hz}$ bandwidth of about $10^{-20}$, $10^{-19}$ and $10^{-19}$, respectively. After the above prototype demonstrations of high strain sensitivities, funding agencies in the US, Europe and Japan committed to the construction of large scale laser interferometers.

There are currently in operation, or in final stages of commissioning, a network of gravitational wave detectors. These detectors operate now with a sensitivity exceeding that of resonant bars, having a larger bandwidth (reaching from a low frequency cut-off at several tens of $\mathrm{Hz}$ up to several $\mathrm{kHz}$ ) and extending the search to a much broader range of potential sources.

The American $\mathrm{LIGO}^{1}$ observatories [20] consist of a $4 \mathrm{~km}$ arm length interferometer in Liv-

\footnotetext{
${ }^{1}$ http: //www.ligo.caltech.edu/
} 


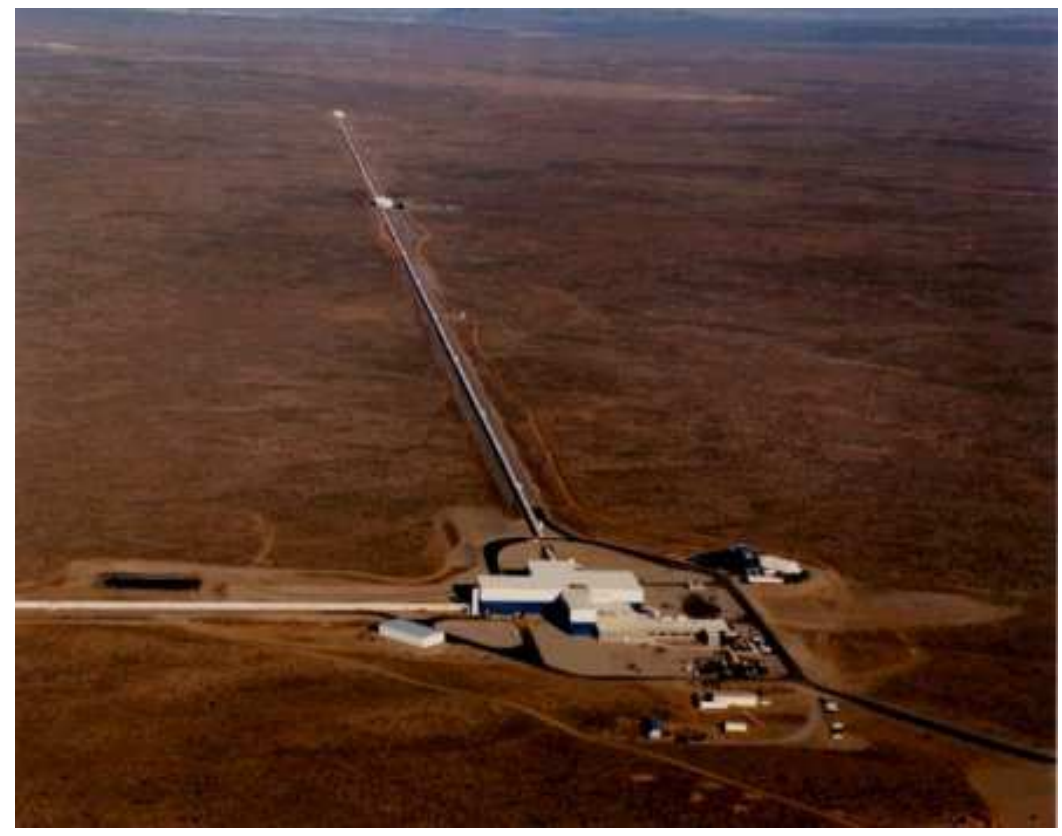

Figure 4: Aerial view of LIGO Hanford site, showing vertex building and orthogonal beam tubes.

ingston, Louisiana, a $4 \mathrm{~km}$ interferometer in Hanford, Washington, and a $2 \mathrm{~km}$ interferometer also at Hanford. The $2 \mathrm{~km}$ and $4 \mathrm{~km}$ interferometers at Hanford are in the same vacuum system. Construction of LIGO began in 1996 and progress has been outstanding with operation at instrumental design sensitivity having been achieved since the fall of 2005 (see figure 5). The $3 \mathrm{~km}$ long French/Italian VIRGO detector [21] near Pisa is close to completing commissioning and the 300 m long Japanese TAMA 300 detector [22] is operating at the Tokyo Astronomical Observatory. The German/British detector, GEO600 [23], through use of novel technologies is expected to reach a sensitivity at frequencies above a few hundred $\mathrm{Hz}$ close to those of VIRGO and LIGO in their initial operation.

During the commissioning phase, work in the detectors was stopped several times to allow for data taking. These "Science Runs" were called S1, S2, S3 and S4, and happened for 17, 61, 70, and 30 days respectively, starting in Aug 23 2002, Feb 14 2003, Oct 312003 and Feb 22 2005, also respectively. All these runs have involved the LIGO detectors, three have involved the GEO detector and two had the TAMA involvement. Their data were analyzed for a variety of gravitational wave signals by a group of researches known as the LIGO Scientific Collaboration ${ }^{2}$ and new upper limits have been set on the strength of gravitational waves from a range of sources: coalescing compact binaries, pulsars, burst sources and a stochastic background of gravitational waves $[24,25,26,27,28,29,30,31,32]$. The fifth science run of the LIGO detectors started on 4th Nov 2005 with GEO having joined for data taking periods from January 2006 and VIRGO from May 2007. This run ended on 1st October 2007 when the LIGO system had one complete year of triple coincidence data from all three of its detectors and being this the longest stretch of

${ }^{2}$ http://www.ligo.org/ 

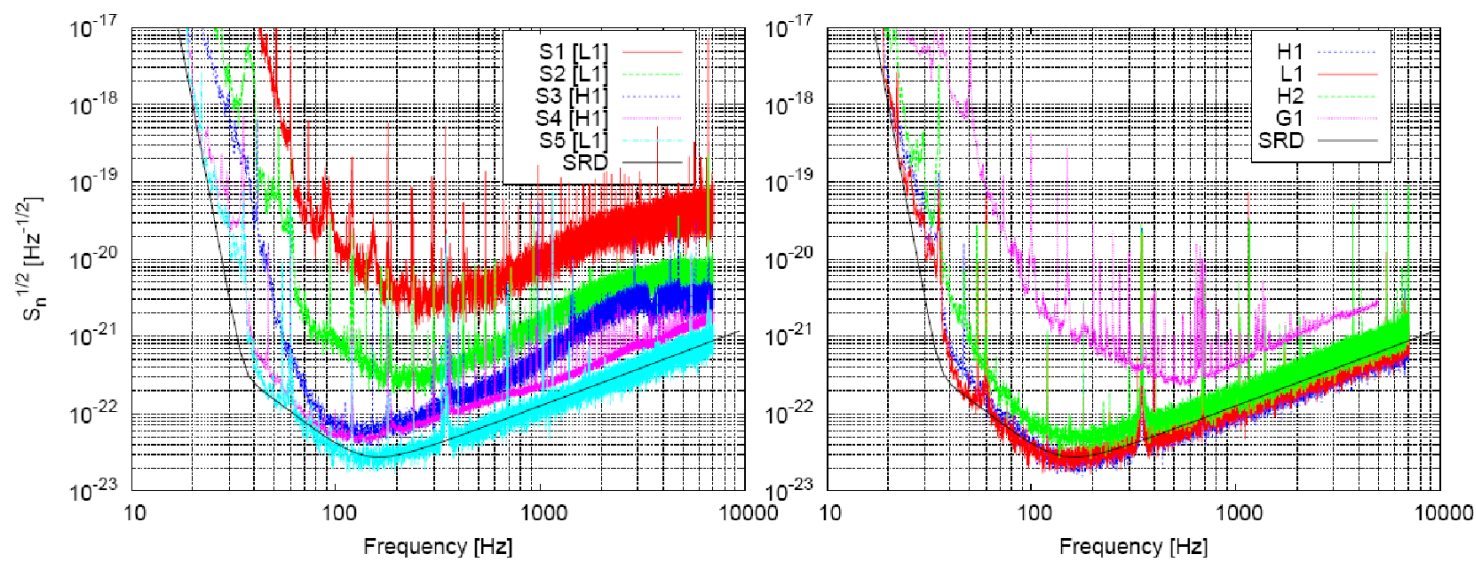

Figure 5: (Left) LIGO instrumental sensitivity for science runs S1 (2002) to S5 (2007) in units of gravitational-wave strain per square root $\mathrm{Hz}$ as a function of frequency. (Right) GEO sensitivity in comparison to the LIGO detectors during S5.

data taking to date at initial design sensitivity.

During the next few years following intermediate upgrades to the LIGO and VIRGO detectors we can expect to see searches for gravitational wave signals at a sensitivity level where a detection could be made. Recent discoveries of additional compact binary systems have improved the statistics for the expected rate of binary coalescences detectable by the LIGO system by a significant factor, giving some possibility of a first detection over the next few years [33]. However detection at the current level of sensitivity of the initial detectors is no way guaranteed, and improvement of the order of a factor of 10 to 15 in sensitivity of the current interferometric detectors is needed to reach sensitivity levels where many signals are expected. Thus plans for an upgraded LIGO, Advanced LIGO, are already well formed. The upgrade is expected to commence in 2010, with full installation and initial operation of the upgraded system by 2014 .

On approximately the same timescale we can expect to see a similar upgrade to VIRGO, the rebuilding of GEO as a detector aiming at high sensitivity in the $\mathrm{kHz}$ frequency region and the building of a long-baseline underground detector, LCGT [34, 35], in Japan. In Australia, the ACIGA consortium operates an $80 \mathrm{~m}$ interferometric testbed and has plans for a future full-scale interferometer.

To go beyond this point, however, a number of challenges involving mechanical losses in coatings, thermal loading effects, and the use of non-classical light to bypass the standard quantum limit will have to be met. Cryogenic test mirrors and non-transmissive optics are likely to be adopted, using materials of high thermal conductivity such as silicon. Thus research groups in the field are already looking towards the next generation of ground based detectors and a European design study for such a system funded under the EC Framework 7 program is expected to commence early in $2008^{3}$.

Close coordination and collaboration between the various detector teams opens the possibility

\footnotetext{
${ }^{3}$ Einstein gravitational wave Telescope, Proposal to the European Commission, Framework Program 7 (2007). http: //www.ego-gw.it/ILIAS-GW/FP7-DS/fp7-DS.htm
} 


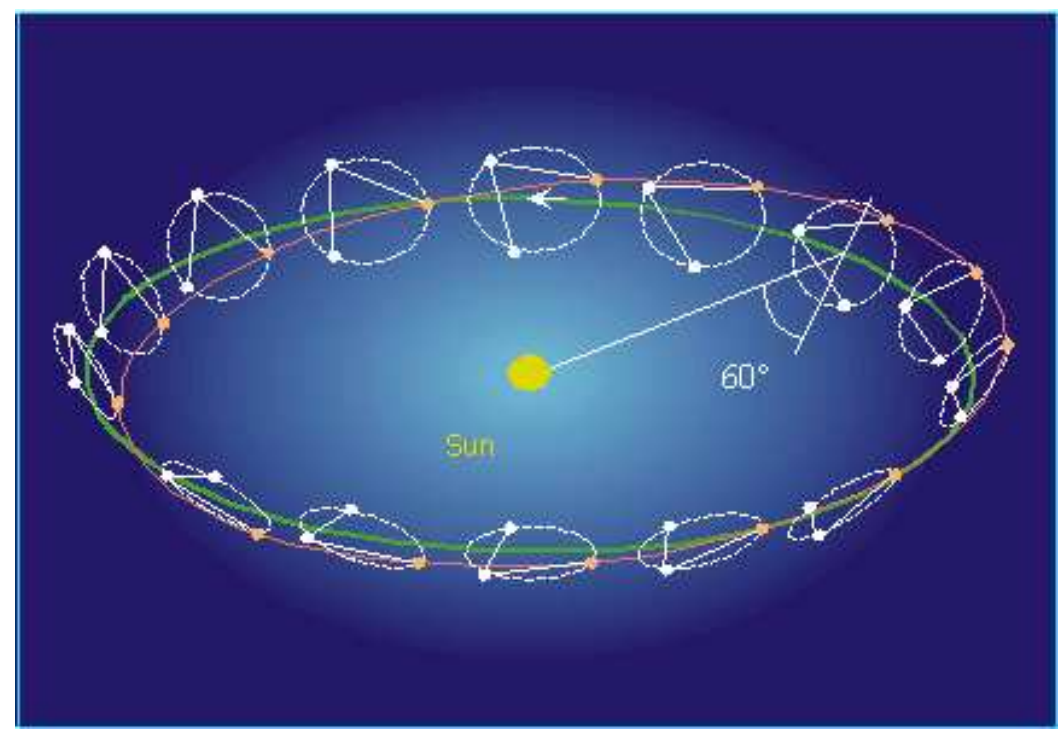

Figure 6: Orbit of LISA constellation. On each spacecraft lasers are phase locked, and each spacecraft transmits and receives light from each of its neighbors. The constellation triangle rotates once per year.

of continuous observation of gravitational waves over the coming years. It also enables an efficient network data analysis. A worldwide network of detectors is essential for achieving a low false alarm rate and locating signal sources in the sky. A global network is a common goal of all projects and is ensured by mutual Memoranda of Understanding that have been signed or are in the process of being signed.

\subsection{LISA: A space-based optical interferometer}

The LISA (Laser Interferometer Space Antenna) Project ${ }^{4}$ [36] is a planned space mission to deploy three satellites in solar orbit forming a large equilateral triangle with a base length of $5 \times 10^{6}$ $\mathrm{km}$. The center of the triangle formation will be in the ecliptic plane 1 AU from the Sun and 20 degrees behind the Earth. Each spacecraft will contain two free-floating proof masses forming the end points of three separate but not independent interferometers. LISA is designed to measure gravitational radiation over a broad band at low frequencies, from about 0.1 to 100 millihertz, a band where the Universe is richly populated in strong sources of gravitational waves. LISA will detect signals from a wide range of different sources: massive black holes merging in galaxies at all distances; massive black holes consuming smaller compact objects; known binary compact stars and stellar remnants; members of known populations of more distant binaries; and probably other sources, possibly including relics of the extremely early Big Bang.

Recently the NASA Beyond Einstein Program Advisory Committee (BEPAC) has recommended that LISA be the Flagship mission of the program, preceded by the Joint Dark Energy Mission (JDEM) and thus LISA is expected to be launched as a joint ESA/NASA mission in 2018 and to be producing data for up to ten years.

\footnotetext{
${ }^{4}$ http://lisa.jpl.nasa.gov
} 


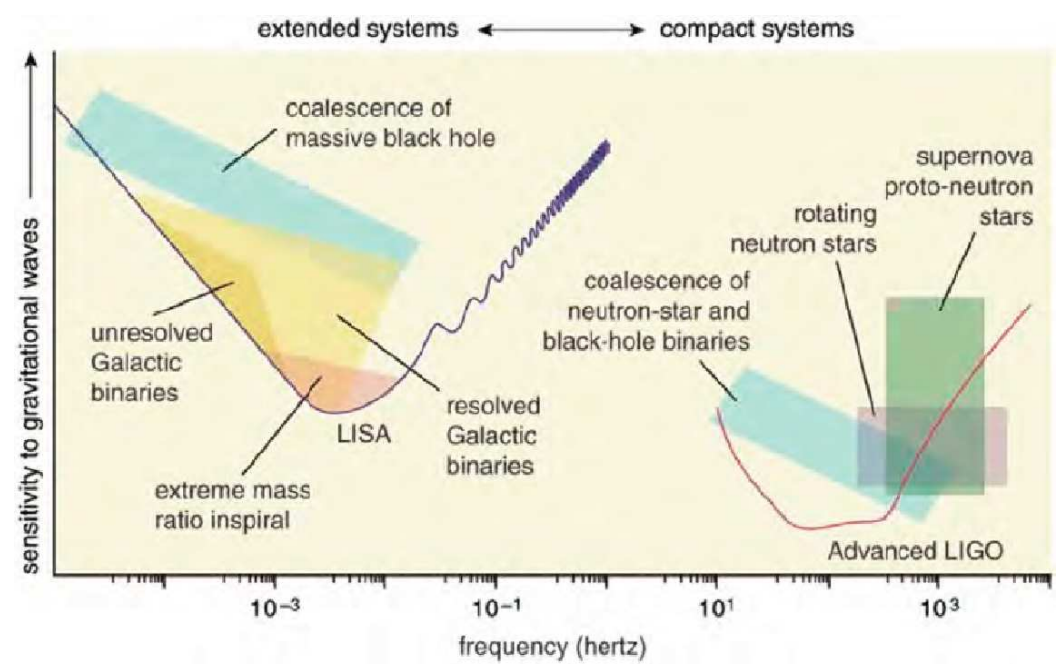

Figure 7: Various possible gravitational wave sources are illustrated along with the expected sensitivities of LISA and Advanced LIGO.

\subsection{Big Bang Observatory: BBO}

$\mathrm{BBO}$ is a follow-on mission to LISA to probe the frequency region of $0.01-10 \mathrm{~Hz}$. Its primary goal would be the study of primordial gravitational waves from the era of the big bang, at a frequency range not limited by the confusion noise from compact binaries. BBO will also extend LISA's scientific program of measuring waves from the merging of intermediate mass black holes at any redshift, and will refine the mapping of space-time around super-massive black holes with inspiraling compact objects. The strain sensitivity of $\mathrm{BBO}$ at $0.1 \mathrm{~Hz}$ is planned to be $\sim 10^{-24}$. This will require a considerable investment in new technology, including $\mathrm{kW}$ power level stabilized lasers, picoradian pointing capability, multimeter sized mirrors with sub-angstrom polishing uniformity, and significant advances in thruster, discharging, and surface potential technology.

\section{Astrophysical sources}

The gravitational waves we expect to observe must be emitted by massive astrophysical objects. An extensive overview of promising sources can be found in [7]. Here we only give a brief summary.

\subsection{Coalescing compact binaries}

Compact binaries are among the most promising sources of gravitational waves for groundbased detectors like LIGO and VIRGO, and the planned space-based detector LISA. Typical examples are the coalescences of binary neutron stars or black holes. Even more spectacular events could be observed from galaxy collisions and the subsequent mergers of massive black holes residing in the centers of the galaxies. A census of a significant portion of the visible Universe would allow us to study the evolution of the population of stars over cosmological time-scales and dynamical interactions in different stellar environments. 
The evolution of binary black hole coalescences is conventionally split into three stages: inspiral, merger and ring down. Gravitational waveforms from the inspiral and ringdown stages can be accurately computed by approximation/perturbation techniques in general relativity [37, 38]. The recent progress in numerical relativity has enabled to model also the merger phase of the coalescence of binary black holes [39, 40, 41] for some particular cases. Combining the results from analytical and numerical relativity will enable to coherently search for all the three stages of the binary black-hole coalescence, which is significantly more sensitive than the current searches [31], and improve the estimation of the parameters of the binary, which is particularly important for LISA.

The observations of massive black hole coalescences will address several of LISA's science objectives. Firstly, detection of the signals from massive black hole binaries themselves will provide direct observations of the black holes. Measuring the spins and masses of the massive black holes will give us valuable information about the mechanism of their formation: rapid spins will imply that much of the black holes mass were built up by gas accretion from a disk, moderate spins imply building the massive black holes by a sequence of major mergers of comparable mass black holes and the low spins imply that massive black holes are mostly built by capturing smaller objects coming from random directions. Knowing the parameter values of the central objects will also enable more accurate studies of the dynamics of the stellar populations in the bulge.

Massive black holes will serve as laboratories for fundamental tests of gravitational theory. The measurement of their masses and spins will confirm (or disprove) some of the untested predictions of General Relativity. We should be able to probe predictions of General Relativity in the different stages of binary evolution starting with a moderately relativistic inspiral phase to a nonlinear strong-field regime. Detecting and characterizing the post-merger phase, where the resulting black hole sheds irregularities and deformations in a well-understood process resulting in ringdown radiation, will allow us to test the 'no-hair' theorem for black holes. In addition, since many of the massive black hole mergers are likely to have electromagnetic counterparts, it is possible to constrain the values of cosmological parameters by combining the gravitational wave and electromagnetic observations [42]. In particular, using the distance-redshift relation from many binary black-hole standard sirens, LISA will be able to put interesting constraints on the equation of state of the dark energy [43]. The error bars on this depend on how accurately the red-shifted mass of the source and the luminosity distance are estimated, and how well the host galaxy of the electromagnetic counterpart is identified. This will have a tremendous impact on one of the outstanding issues of present-day cosmology.

\subsection{Galactic Binaries}

Low-mass binaries (also known as compact stellar-mass or ultra-compact binaries) are binary systems containing white dwarfs, naked helium stars, neutron stars or black holes. LISA will be able to detect such binaries if they are located within our Galaxy, in the surrounding globular clusters. The most common sources are expected to be white-dwarf binaries emitting gravitational wave signals of nearly constant frequency and amplitude.

Verification binaries are the systems known from previous astronomical observations. We know sky positions and approximate periods, distances, and masses for many of these sources. 
Observation of these known binaries will provide a check of the operation of the instrument, as well as a strong test of General Relativity.

It is expected that LISA will detect and characterize around 10000 individual compact binary systems, as well as establish an accurate estimate of the stochastic foreground produced by tens of millions of binaries in our Galaxy. The resolved systems will provide a unique map of ultracompact binaries. The gravitational wave measurements of this population, largely inaccessible to electromagnetic detectors, will provide information about the formation and evolution of compact binaries in general and the physics of mass transfer and tides in white dwarfs in particular. In addition, up to $10 \%$ of the white dwarfs systems observed by LISA will be seen by optical followups, allowing for interesting cross-comparisons and tests of fundamental physics. For example, by comparing the arrival times of the gravitational and electromagnetic signals it will be possible to place improved bounds on the mass of the graviton [45].

\subsection{Massive black hole captures}

Supermassive black holes at the center of galaxies regularly capture objects from the clouds of stars surrounding them. Many of these are compact stars and black holes that have, by chance, been deflected onto orbits close enough to the black hole for their first encounter with the hole to place them into a bound orbit, through the loss of energy to gravitational radiation. Other objects may have been captured by tidal effects: compact-object binaries that have come too close to the hole and been disrupted by tidal forces; compact cores of giant stars that have been tidally stripped by the central hole; or black holes that have formed near the central hole by the evolution of giant stars such as those seen near the central hole in the Milky Way. The resulting compact objects (neutron stars, black holes, white dwarfs) would then survive as point-like objects orbiting the central hole until their losses to gravitational radiation send them across the horizon.

EMRIs are Extreme Mass Ratio Inspiral sources, where the ratio of the mass of the inspiraling object to that of the central black hole is $10^{-4}$ or smaller. Because they are more massive, black holes sink to the center of star clusters and will be over-represented among the EMRI population. Also because of their mass they can be seen further away than neutron-star or white-dwarf captures, so the typical expected system is a stellar black hole with a mass of 10 (solar mass) into a $10^{6}$ (solar mass) supermassive black hole.

EMRIs figure among the principal fundamental-physics goals of the LISA mission because their signals contain rich information about the geometry around the central black holes. The phase evolution of their signals, lasting for thousands or even hundreds of thousands of cycles, reflect in detail the near-geodesic orbits they follow around the holes. From this phase information we expect to measure how closely the geometry matches the Kerr geometry predicted by general relativity [44], and thereby test the black-hole uniqueness theorems of Einstein's theory. Direct evidence of the existence of a horizon in the spacetime will come from seeing the signals cut off as they cross the horizon. If they do not cut off, then that will indicate that the central object is not massive black hole; explaining what it is will require exotic new physics.

\subsection{Stellar collapse, supernovae and gamma-ray bursts}

The collapse of a massive star, after gravitation overwhelms the pressure sustained through nuclear burning, results in a supernovae explosion and the remnant in a neutron star or black hole. 
The core collapse, if it is sufficiently asymmetric, has sufficient mass dynamics to be a source of gravitational waves. However the physics of the process from collapse to compact object formation is not well understood and such events are rare (several per century per galaxy). This violent explosion will produce a burst signal, one that is short in time, but relatively large in amplitude.

Other possible sources may also produce intense gravity signals that are short in time. In addition to supernovae, there could be burst-like signals from the final merger of neutron star or black hole binary systems, instabilities in nascent rotating neutron stars, or kinks and cusps on cosmic strings. Unfortunately, the exact gravitational waveform for most of these types of events are not well-known (the string waveform is, in fact, known), so general burst search templates need to be employed [29, 30].

Coalescing compact binaries are currently believed to be sources of short gamma-ray bursts, and supernova explosions to be the sources of long gamma-ray bursts. The observation of the gravitational waves expected from such events will contribute to a better understanding of the processes leading to gamma-ray bursts. Particularly interesting would be coincident observations of neutrinos and gravitational waves from supernovae and gamma-ray bursts.

\subsection{Spinning neutron stars}

Rapidly spinning neutron stars, or pulsars, are the other key targets in the high-frequency band: they are cosmic laboratories of matter under extreme conditions of density, temperature and magnetic fields. The gravitational wave detectors will open a radically new window to explore such phenomena.

Although any departure from axial symmetry in a spinning neutron star will result in gravitational radiation, and despite having a fair idea of the neutron star population in the galaxy, we have little idea how smooth they are and therefore whether any will be visible with the current generation of detectors. We can however make a fair guess at how mountainous they are: the spin down rates of pulsars (radio or X-ray loud spinning neutron stars) are easily observed. The spin down must be at least partially due to magnetic dipole braking, as pulsars are highly magnetized objects, but if we allocate all we see to gravitational radiation we can, with some assumptions, place an upper limit on the likely mass quadrupole of each pulsar. Doing this, it seems unlikely that more than two or three known radio pulsars could be seen right now, though more extensive searches are ongoing [26]. The most likely candidate is the Crab pulsar, whose spin-down limit promises to be well and truly broken with current observational sensitivities.

Using the LIGO S5 data, limits on the strain signal strength as low as $h_{0} \sim 4 \times 10^{-26}$ are achieved for some pulsars. This corresponds to limits on pulsar ellipticity as low as $10^{-7}$. Searches for gravitational waves from radio quiet neutron stars are more difficult and indeed represent a massive computational challenge [24, 32]. This has been met in part by the Einstein@Home project ${ }^{5}$, a BOINC-based screensaver actively running on around 100000 computers worldwide and currently delivering about 80 teraflops of processing power to the problem.

\subsection{Stochastic background}

Finally, a variety of cosmological scenarios predict a cosmological background of gravita-

\footnotetext{
${ }^{5}$ http://einstein.phys.uwm.edu
} 
tional waves, analogous to the electromagnetic cosmic microwave background. This would seem to be a background noise in each detector, but the signal could be extracted through a correlation of the outputs of two or more independent detectors (see for example [25, 27, 28]). Measuring the spectrum of the stochastic background would connect us to the Planck era and would be a good mean to discriminate the different cosmological models: inflation, excitations of scalar fields arising in string theories, QCD phase transitions, coherent excitations of our universe, regarded as a brane in a higher dimensional universe, etc. However, for most models the predicted amplitude of the stochastic background is well below the sensitivity of the current LIGO detectors. If detectable will open a new window to probe fundamental physics processes in regions and at energy scales hitherto not accessible.

\section{Acknowledgments}

I would like to thank the organizing committee for the invitation to give this talk. I am also grateful to colleagues in GEO600 and the LIGO Scientific Collaboration for help, and the support by the Spanish Ministerio de Educación y Ciencia research projects FPA-2007-60220, HA20070042, and the Conselleria d'Economia Hisenda i Innovació of the Government of the Balearic Islands.

\section{References}

[1] Einstein, A. 1916, Preuss. Akad. Wiss. Berlin, Sitzungsberichte der physikalish-mathematischen Klasse, 688

[2] Einstein, A. 1918, Preuss. Akad. Wiss. Berlin, Sitzungsberichte der physikalish-mathematischen Klasse, 154

[3] Hulse, R. A. and Taylor, J. H. 1974, Astrophys. J. 191, L59

[4] Hulse, R. A. and Taylor, J. H. 1975, Astrophys. J. 195, L51

[5] Hulse, R. A. and Taylor, J. H. 1975, Astrophys. J. 201, L55

[6] Weisberg, J.M. and Taylor, J.H. 2005, in “Binary Radio Pulsars”, eds. F.A. Rasio \& I.H. Stairs (Proc. Aspen Conference, ASP Conf. Series, Vol 328, p.25, USA) [arXiv: astro-ph/0 407149]

[7] Thorne, K.S. 1987, in “300 Years of Gravitation”, ed. S.W. Hawking \& W. Israel (Cambridge U. Press: Cambridge, UK)

[8] Hough, J., Rowan, S. and Sathyaprakash, B.S. 2005, J. Phys. b: At. Mol. Opt Phys. 38, S497

[9] Weber, J. 1960, Phys. Rev. 117, 306

[10] Weber, J. 1969, Phys. Rev. Lett. 22, 1320

[11] Baggio, L. et al. 2005, Phys. Rev. Lett. 94, 241101

[12] Astone, P. et al. 2006 Class. Quantum Grav. 23, S57

[13] Heng, I. S. et al. 2001, Class. Quantum Grav. 19, 1889

[14] Moss, G.E., Miller, L.R. and Forward, R.L. 1971, Appl. Opt. 10, 2495

[15] Weiss, R. 1972, Quarterly Progress Report, MIT Research Lab of Electronics 105, 54 
[16] Forward, R.L. 1978, Phys. Rev. D 17, 379

[17] Abramovici, A. et al. 1996, Phys Lett A 218, 157

[18] Robertson, D.I. et al. 1995, Rev. Sci. Instr. 66, 4447

[19] Shoemaker, D. et al. 1988, Phys. Rev. D 38, 423

[20] Abbott, B. et al. 2004, Nucl. Instrum. Meth. A517, 154

[21] Acernese, F. et al. 2006, Class. Quantum Grav. 23, S63

[22] Takahashi, R. TAMA Collaboration 2004, Class. Quantum Grav. 21, S403

[23] Willke, B. et al. 2004, Class. Quantum Grav. 21, S417

[24] Abbott, B. et al. 2007, Phys. Rev. D. 76, 082001

[25] Abbott, B. et al. 2007, ApJ 659, 918

[26] Abbott, B. et al. 2007, Phys. Rev. D 76, 042001

[27] Abbott, B. et al. 2007, Phys. Rev. D 76, 022001

[28] Abbott, B. et al. 2007, Phys. Rev. D 76, 082003

[29] Abbott, B. et al. 2007, Phys. Rev. D 76, 062003

[30] Abbott, B. et al. 2007, Class. Quantum Grav. 24, 5343

[31] Abbott, B. et al. 2007, arXiv:0704.3368

[32] Abbott, B. et al. 2008, Phys. Rev. D 77, 022001

[33] Kalogera, V. et al. 2004, Astrophys. J. 601, L179

[34] Kazuaki, K. and the LCGT Collaboration 2006, Class. Quantum Grav. 23, S215

[35] Kazuaki, K. and the LCGT Collaboration 2006, ApJ (Letters) 447, L95

[36] Danzmann, K and Rüdiger, A. 2003, Class. Quantum Grav. 20, S1

[37] Blanchet, L., Damour, T., Esposito-Farese, G. and Iyer, B. R. 2004, Phys. Rev. Lett. 93, 091101

[38] Teukolsky, S. and Press, W. 1974, Astrophys. J. 193, 443

[39] Baker, J.G., Centrella, J., Choi, D.I., Koppitz, M. and van Meter, J. 2006, Phys. Rev. Lett. 96, 111102

[40] Campanelli, M., Lousto, C.O., Zlochower, Y. and Merritt, D. 2007, Astrophys. J. 659, L5

[41] González, J. A., Sperhake, U., Brügmann, B., Hannam, M. and Husa, S. 2007, Phys. Rev. Lett. 98, 091101

[42] Schutz, B. F. 1986, Nature 323, 310

[43] Holz, D. E. and Hughes, S. A. 2005, Astrophys. J. 629, 15

[44] Glampedakis, K. and Babak, S. 2006, Class. Quantum Grav. 23, 4167

[45] Cutler, C., Hiscock, W. and Larson, S. 2003, Phys. Rev. D 67, 024015 